ESJ Natural/Life/Medical Sciences

\title{
Dose Variation To Critical Points In Low Dose Rate Intracavitary Brachytherapy Of Cervical Cancer As Justification For Incident Learning
}

\author{
Eric C.D.K. Addison,
}

Department of Physics, Kwame Nkrumah University of Science and

Technology, Kumasi, Ghana

Oncology Directorate, Komfo Anokye Teaching Hospital, Kumasi, Ghana

Department of Medical Physics, Graduate School of Nuclear and Allied

Sciences, University of Ghana

Joseph Adom,

Department of Physics, Kwame Nkrumah University of Science and

Technology, Kumasi, Ghana

Oncology Directorate, Komfo Anokye Teaching Hospital, Kumasi, Ghana

\section{Francis Hasford,}

John H. Amuasi,

Department of Medical Physics, Graduate School of Nuclear and Allied Sciences, University of Ghana.

Doi:10.19044/esj.2022.v18n03p48

Submitted: 22 November 2021

Accepted: 26 December 2021

Published: 31 January 2022
Copyright 2022 Author(s)

Under Creative Commons BY-NC-ND 4.0 OPEN ACCESS

Cite As:

Addison E.C.D.K., Adom J., Hasford F., \& Amuasi J.H., (2022). Dose Variation To Critical Points In Low Dose Rate Intracavitary Brachytherapy Of Cervical Cancer As Justification For Incident Learning European Scientific Journal, ESJ, 18 (3), 48.

https://doi.org/10.19044/esj.2022.v18n03p48

\section{Abstract}

Background: Low Dose Rate (LDR) brachytherapy necessitates a more extended treatment duration. This technique maintains the patient on the treatment machine for a longer length of time, which may cause changes in the applicators' position due to vaginal packing soaking and patient movement for incident learning justification.

Objective: The purpose of this study is to evaluate pre- and postdosage variation to points 'A' and 'B,' as well as vital organs (i.e. bladder and rectum), for low dose rate (LDR) brachytherapy at Komfo Anokye Teaching Hospital and justify the need for incident learning system. 
Method: Forty (40) patients with invasive cervical cancer were treated with LDR brachytherapy equipment (A Curietron Cesium Manuel (AMRA)France, CA 98.22) to point 'A' at doses ranging from 30 to 35 Gy. Adult patients selected for the study ranged from 25 to 60 years simulated. For each patient, orthogonal images of anterior-posterior (AP) and (LAT) were taken by positioning the patient on the Varian Acuity Simulator Couch at 00 and 900, respectively, and two sets of orthogonal images (before and after treatment), in each case, were considered. The treatment was then planned using the AP and LAT images obtained based on the four arrangements used, namely $1-2-5,1-3-5,1-4-5$, and $1-5$. The treatment planning system used for the study was the Prowess Panther system 4.6.

Results: The dosage disparity at point 'A' was determined to be 1.16 per cent, which is highly commendable compared to previous research that established dose variations of $2 \%, 35 \%, 8 \%$, and $20 \%$. In this study, the dosage variation at point B was 0.75 per cent. The bladder and rectum had average alterations of 2.32 per cent and 0.30 per cent, respectively.

Conclusion: The difference observed between prescribed, and deposited dosage was 2.11 per cent for quality assurance and incident learning reasons. Unrealistic expectations, reliance on reminders and quick remedies, and faults in the systemic view of failure were discovered and addressed as managerial issues. It is critical not to underestimate these difficulties because they are frequently significantly more severe than the technical issues being addressed. Incident issues were not recorded per patients under review, but near misses and non-conformance were observed. The geometric variances between the Intracavitary Brachytherapy Treatment (ICBT) applicators and the essential organs change during the treatment process, resulting in dosage changes. Does variance is within the required standard limits; it can be stated that Komfo Anokye Teaching Hospital's practice satisfies the international standard and is an intrusion into the Directorate Incident Learning System.

Keywords: Low dose rate (LDR) Brachytherapy (BT), Cervical cancer, Point A, Point B, Intracavitary Brachytherapy Treatment

\section{Introduction}

Cancer is a disease that occurs at the cellular deoxyribonucleic acid (DNA) level. It is an uncontrolled growth of human cells, spreading to surrounding and remote tissues. Cancer can invade other body parts through the hematopoietic and lymphatic systems. There are over one hundred types of cancers and are mostly named by the organ or kind of cell they attack (cancer that begins in melanocytes of the skin is melanoma). Cervical cancer begins in cells lining the cervix. The squamous cells and glandular cells are 
the two main cells covering the cervix. The meeting place of these types of cells is known as the transformation zone. Early detection of the tumour makes its treatment quite easier and effective. If there is uncontrolled cell growth, it will end up in the individual's death (Chaffer et al., 2011). The past years have observed a great development towards treating and understanding the previously proposed cancer hallmarks (Hanahan and Weinberg, 2000). With improvements in early detection and the different treatment modalities, many cancers have become curable (Pollack et al., 2009). Cervical cancer is the fourth most occurring cancer affecting women globally, after breast, colorectal, and lung cancers, with 528000 new cases every year; it is most common in the lower resource countries of sub-Saharan Africa (WHO, 2012). Cancer of the cervix is responsible for the death of an estimated 231000 women annually, with over $80 \%$ of these deaths occurring in developing countries (Domfeh et al., 2008). In Ghana, mortality due to cervical cancer is on the rise due to the absence of an organised and effective cervical cancer prevention and control program (Adams, Nortey, Dortey, Asmah, \& Wiredu, 2019). The second most prominent female malignancy in sub-Saharan Africa is cervical cancer (Parkin et al., 2003). Southern and Eastern Africa, with respectively 43 per 100000 women and 37 per 100000 women, reported the maximum incidence rates in the world (WHO, 2008). The most common cancer type varies among countries. Certain cancers, such as cervical cancer, are much more common in countries at the lower end of the human development index (HDI) than in high-HDI countries. Incident cases of 569 847 for Cervix uteri (WHO, 2020). The corresponding mortality rates are 34 per 100000 women and 23 per 100000 women. For instance, the incidence rate for all races is 8.2 per 100000 women in the United States (Leaver and Labonte, 2010), while Makin and Kamanu (2010) noted that mortality rates in resource-rich countries seldom exceed 5 per 100,000 women. This difference between Africa and the advanced world is enormous, and the success in the latter is attributed largely to widespread comprehensive cervical cancer screening control programs (WHO, 2008; Adewole et al., 2005). In Africa, with 267.9 million women aged 15 years or greater, estimates are that 78897 women are diagnosed with cervical cancer annually, and 61671 (78\%) die from the disease (Denny, 2010). Radiotherapy, in the form of External Beam Radiotherapy (EBRT), brachytherapy or both, constitutes an essential aspect of treating carcinomas. Cancers such as those of the breast, prostate, cervical 
and skin are usually treated by combining EBRT and brachytherapy. In Ghana, brachytherapy is usually used to manage cervical and sometimes prostate cancers. Margaret Cleaves first performed intracavitary brachytherapy (ICBT) in 1903, which involves placing uterine tandem and vaginal ovoids (Koushik et al., 2010). The three types of brachytherapy machines or processes developed are high dose rate (HDR), medium dose rate (MDR) and low dose rate (LDR) brachytherapy. In using LDR brachytherapy, the displacement and motion of the applicator as a result of prolonged treatment time and unavailability of rigid applicator fixation is one of the most major problems (Ljunggren et al., 1987). In some cases, only two ovoids are used when the patient has undergone total or partial hysterectomy. Low dose rate intracavitary brachytherapy involves the delivery of radiation at a continuous rate of $0.4-2 \mathrm{~Gy} / \mathrm{hr}$, and this means delivering 30 to $35 \mathrm{~Gy}$ will take around 30 to 50 hours. Due to this long treatment time, soakage of vaginal packing and patient movement can change the position of the applicators. These changes go a long way to defeat the intent of the oncologist and make most of the patients have some complications such as fistula after treatment. Given this, there is the need to evaluate the dose variation to the critical point and organs due to the prolonged treatment time, hence, the justification for incident learning and safer radiotherapy. This is achieved by evaluating the geometric movement of the fixed reference points in LDR brachytherapy patients and determining the actual dose difference, in turn to critical structures and the clinical outcome. Incident Reporting and Learning System (IRLS) is a unique, empirical safety mechanism in radiotherapy practice. It thoroughly addresses patient and personnel protection and high-quality practice in the Radiotherapy setting in all facets of the radiotherapy community as a necessary condition for safer radiotherapy care. In the Departments of Radiation Oncology, IRLS is gaining the momentum needed to be used as a precondition for improving quality health care and patient safety. Numerous organisations such as Safety in Radiation Oncology (SAFRON) system, the Radiation Oncology Incident Learning System (RO-ILS) system, the National System for Incident Reporting (NSIR-RT), and the Radiotherapy Incident Reporting and Review System (RIRAS) advise on this (Kohn, Corrigan, and Donaldson, 2000). As a result of advances in treatment techniques, and advanced irradiation equipment, radiotherapy has evolved into a scientific discipline involving personnel from various backgrounds, including radiation oncologists, medical 
physicists, biomedical engineers, quality experts, and radiation therapy technologists.

\section{Material And Methods}

In this study, materials used for patient set up and absorbed dose calculations include Fletcher type brachytherapy applicators. Others are the Varian Acuity simulator, Prowess Panther system 4.6 treatment planning system (TPS) and Cs-137 Curietron (AMRA) Fletcher- France (CA 98.22) LDR brachytherapy system. The age group of adult patients for the study was between 25 and 60, all females with the tumour stage I-IV at KATH. Orthogonal images of anterior-posterior (AP) and lateral (LAT) were taken for every patient using the Varian Acuity simulator. In each case, two sets of orthogonal images (before and after treatment) were considered to decide various parameters relating to the applicator geometry and its spatial relation to the bony pelvis. In order to see the bladder and rectum, Urografin (radioopaque substance) was passed through a catheter into the organs. The gauze was packed into the vagina in order to stabilise the applicators. Reference planes $(\mathrm{x}, \mathrm{y}, \mathrm{z})$ were also defined for each set of images by using the patient's bone landmarks to evaluate changes in the positioning of applicators relative to fixed bony landmarks of the patient. The exact patient positioning for the post-treatment images was identified with marks on the patients.
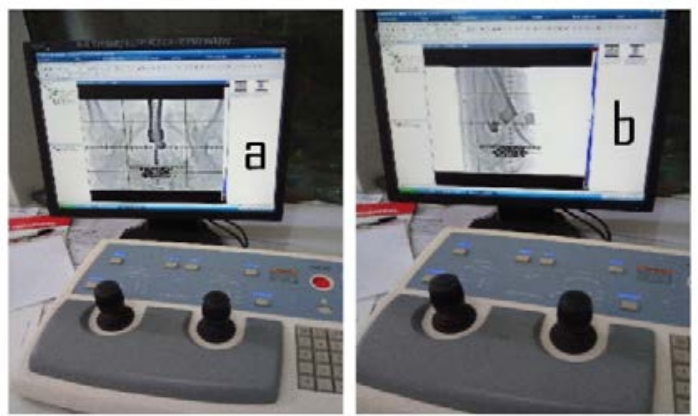

Figure 1: (a) AP image of the pelvis of a patient (b) LAT image of the pelvis of a patient

The Curietron LDR brachytherapy treatment machine has five channels where the ovoids (marked ' $\mathrm{V}$ ') and tandem (marked 'U') connect. During treatment, only one channel of the "U" was selected and used depending upon the measured length of the tandem that protruded. It has been found that channel two was required to be used if the protruded tandem's measured length (L) is $\leq 4 \mathrm{~cm}$. Alternatively, if the measured length of the protruded tandem was between 4 and $5 \mathrm{~cm}$, then channel three was required to be used. If the 
measured length of the protruded tandem was found to be $>5 \mathrm{~cm}$, then channel four was required to be used. This study used four source arrangements or channel combinations, namely 1-2-5, 1-3-5, 1-4-5, and 1-5. The channel 1-5 combination was used when the patient had undergone total or partial hysterectomy. After the various combinations, the Cs-137 radioactive sources in the brachytherapy system were called in (moved into treatment positions) from their respective storage locations. The time at which the sources were called from its safe was noted.

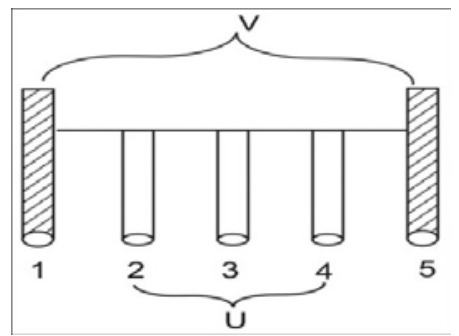

Figure 2: A schematic diagram of channel arrangement in AMRA-Curietron intracavitary brachytherapy system

The treatment was planned using the AP and LAT images obtained. The outline shape of the ovoid (on the lateral image) was obtained, and the centre was located. The new diameters of the ovoid and tandem on the image were measured (I), with the original diameter of $20 \mathrm{~mm}$ and $6 \mathrm{~mm}$ for the ovoid and tandem, respectively. Equation 1 was used to estimate the magnification of the images (given tandem $\mathrm{d}_{\text {tandem }}=6 \mathrm{~mm}, \mathrm{~d}_{\text {ovoid }} 20 \mathrm{~mm}$ ).

$$
\begin{array}{ll}
\text { Magnification }(M)=\frac{\text { Image size }}{\text { object size }} & 1 \\
M_{\text {tandem }}=\frac{I}{6} \quad M_{\text {ovoid }}=\frac{I}{20} & 2
\end{array}
$$

The average of the magnifications of ovoid and tandem was then estimated and taken as the magnification factor of the lateral image $M_{\text {lat }}$.

$$
M_{\text {lat }}=\frac{M_{\text {tandem }}+M_{\text {ovoid }}}{2}
$$

The active source length $(17.2 \mathrm{~mm})$ was then multiplied by the image magnification, to obtain the active source length which was used for the treatment planning.

$$
\text { source length }_{\text {apparent }}=M_{\text {lat }} \times 17.2 \mathrm{~mm} \quad 4
$$

The centre of the tandem was identified and the sources were allocated on it using a pair of vernier calipers including the interval between sources (given actual interval $3.1 \mathrm{~mm}$ ).

$$
\text { interval }_{\text {apparent }}=M_{\text {lat }} \times 3.1 \mathrm{~mm} \quad 5
$$

A perpendicular line was drawn through the last point of the line source. One active source length () was subtracted from ovoid length (apparent), then the 
average was found. A vernier calliper was used to demarcate the points from both ends of the ovoid. The centre between two sources was noted, and a perpendicular line was drawn parallel to the perpendicular line drawn through the last point source. Point ' $A$ ' was located $2 \mathrm{~cm}$ above the intersection of the ovoid, and the perpendicular line passing through the centre of the source was located in the ovoid and traced adjacent to the tandem. Point ' $\mathrm{B}$ ' was located $3 \mathrm{~cm}$ away from point A lateral to the tandem on the AP image. Both points ' $A$ ' and ' $B$ ' were located on the image as multiples of the image magnification.

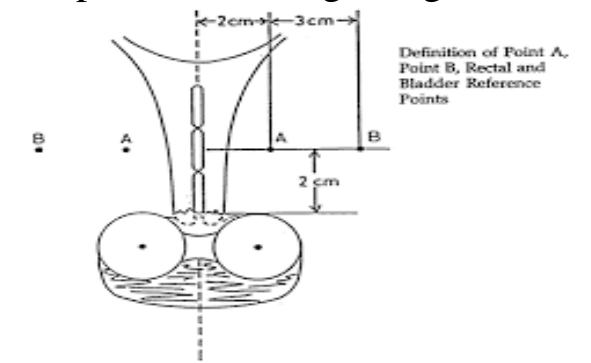

Figure 3: The position of dosage points A and B.

The LAT and AP X-ray images were digitized to generate a plan for treatment delivery. Beginning with the lateral, then to the AP, the points on the images were transferred unto the TPS. The absorbed dose to tissue/medium is given by,

$$
\begin{array}{ll}
D_{\text {med }}=D_{\text {air }}\left(\frac{\mu_{e n}}{\rho}\right)_{\text {air }}^{\text {med }} & 6 \\
D_{\text {med }}=X\left(\frac{W_{\text {air }}}{e}\right)\left(\frac{\mu_{\text {en }}}{\rho}\right)_{\text {air }}^{\text {med }} & 7
\end{array}
$$

Where, $\left(\frac{W_{\text {air }}}{e}\right)=\frac{33.97 \mathrm{~J}}{C} ;\left(\frac{\mu_{\text {en }}}{\rho}\right)$ is the mass energy absorption coefficient, and $\mathrm{X}$ been exposure rate in the air. The mathematical equation for the treatment time is given as;

where $\mathrm{D}=$ Prescribed dose,

$$
t=\frac{D}{\dot{D}}
$$

$\dot{D}=$ Dose rate of cesium- 137

$\mathrm{D}_{\text {med }}=$ absorbed dose to tissue/medium,

$\mathrm{D}_{\text {air }}=$ absorbed dose in air,

$\mu_{\text {en }}=$ energy absorption coefficient,

$\rho=$ density of the medium 


\section{Results}

Variability in doses to points 'A', 'B', bladder, and rectum resulting from change or movement of applicators have been studied for forty (40) patients. Thirty-four (34) of the patients had their cervix present, whiles six (6) patients had undergone hysterectomy. The variations were studied in (a) difference in pre and post-treatment calculated doses and; (b) difference in prescribed and delivered doses.

The dose variations at point 'A' for 34 patients with their cervix present is shown in Figures 4, 5, and 6 below;

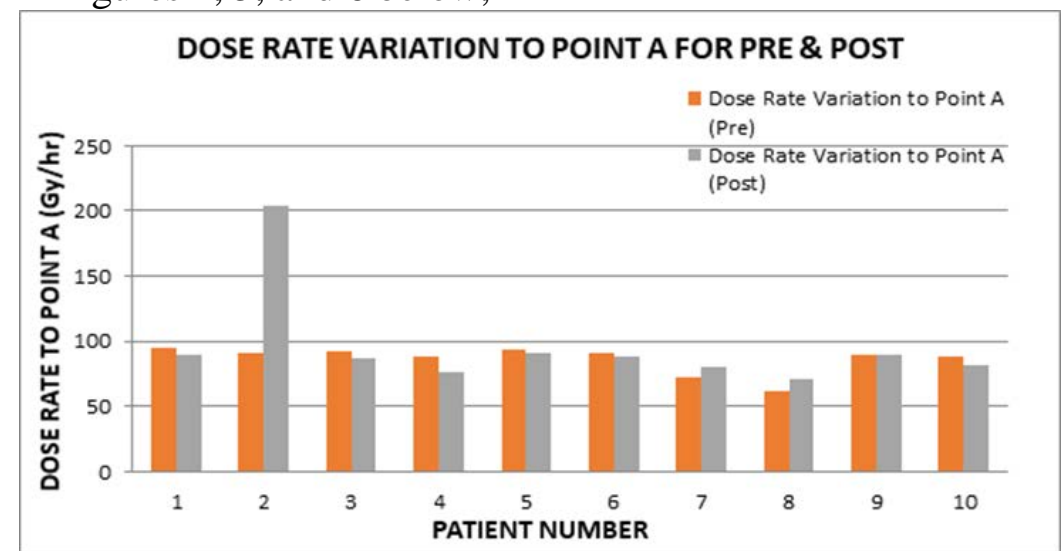

Figure 4: A bar chart of dose rate variation to point 'A' for pre \& post-treatment for first 10 patients

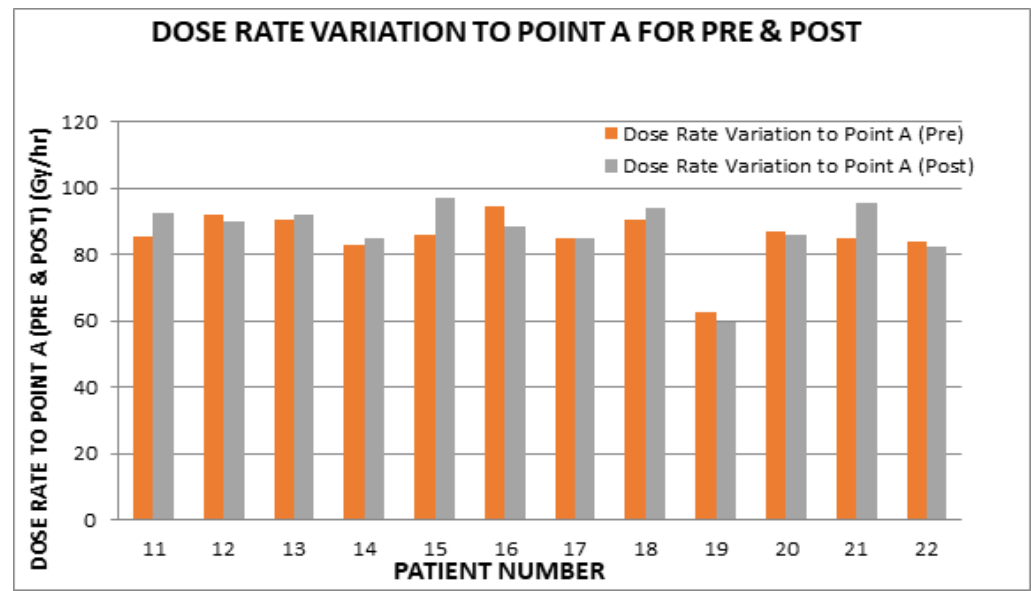

Figure 5: A bar chart of dose rate variation to point 'A' for pre \& post-treatment for the next set of 12 patients 


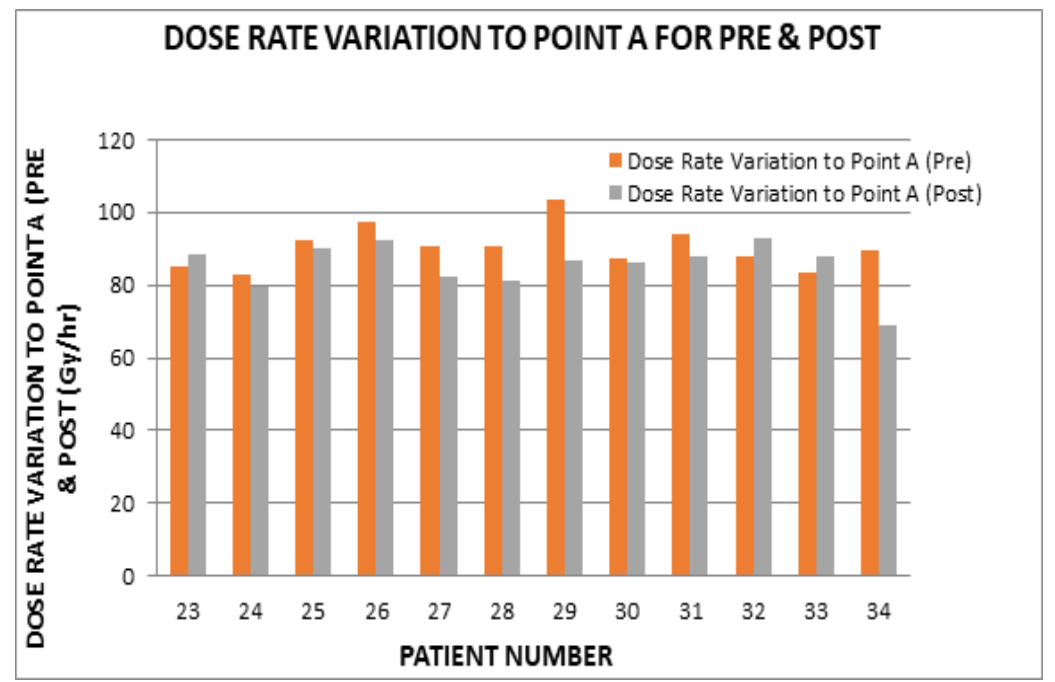

Figure 6: A bar chart of dose rate variation to point 'A' for pre \& post-treatment for the last 12 patients

Figure 7 show the dose variation at point A for 6 patients who had undergone hysterectomy

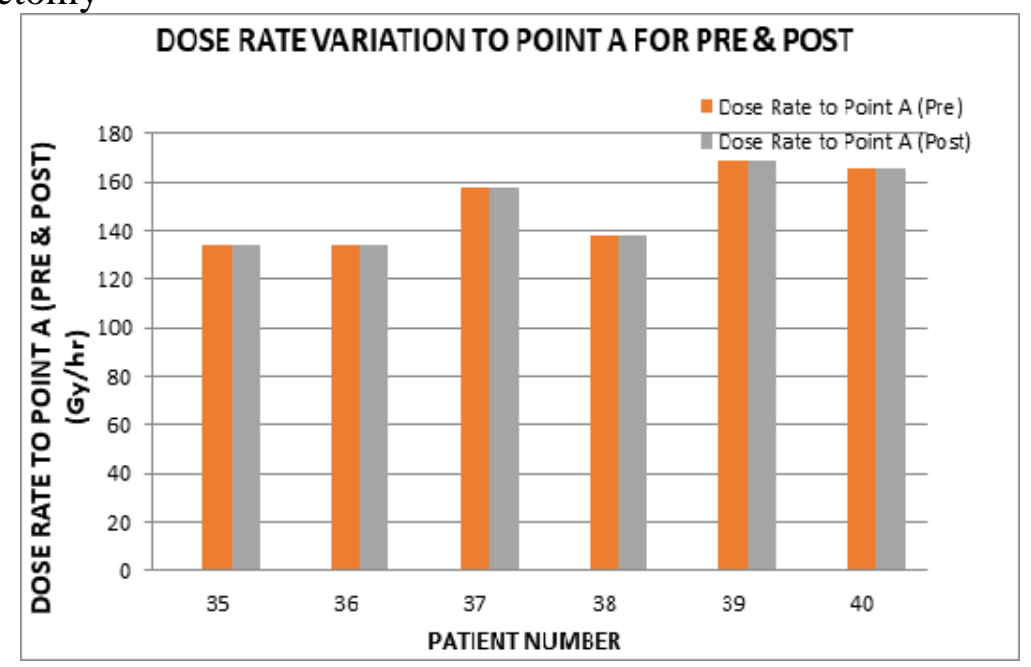

Figure 7: A bar chart of dose rate variation to point 'A' for pre \& post-treatment for 6 hysterectomy patients

The dose prescribed and received for 40 patients are as below 


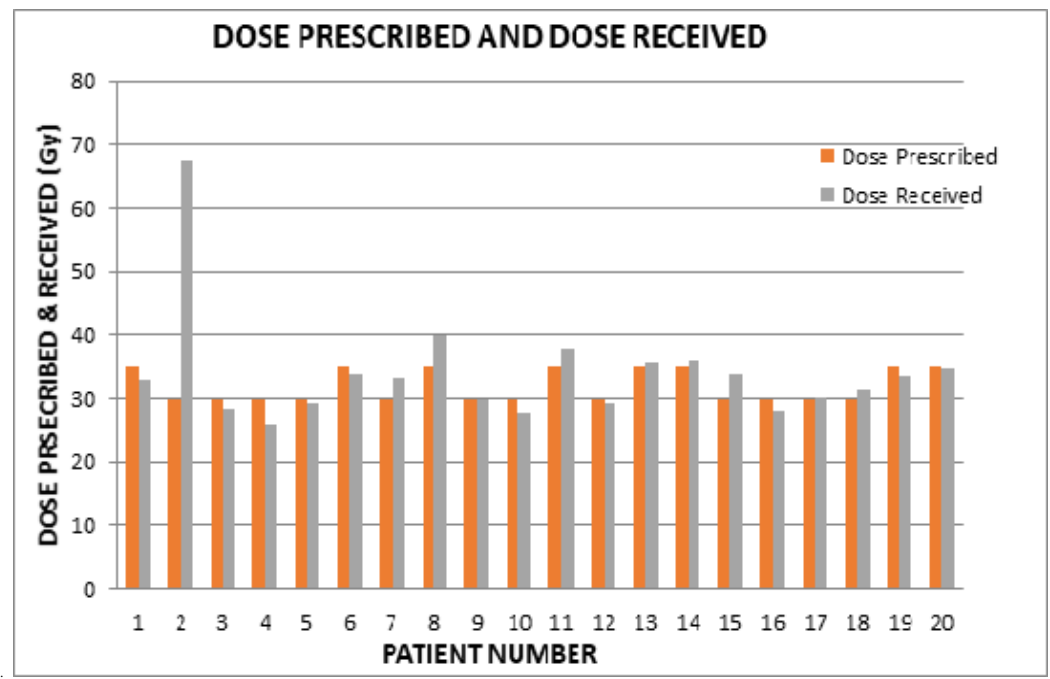

Figure 8: A bar chart of dose prescribed and received for the first 20 patients

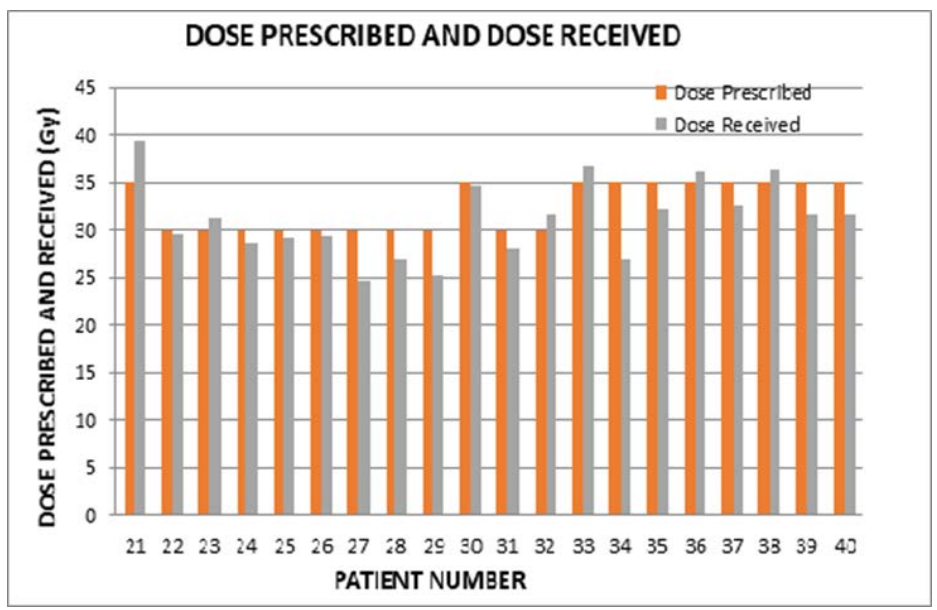

Figure 9: A bar chart of dose prescribed and received for the last 20 patients

Point ' $B$ ' is $5 \mathrm{~cm}$ lateral from the midline at the same level as point ' $A$ '. The dose variations at point ' $\mathrm{B}$ ' for 34 patients with their cervix present is shown in Figures 10, 11, and 12; 
Figure 10: A bar chart of dose rate variation to point 'B' for pre \& post-treatment for first 10 patient
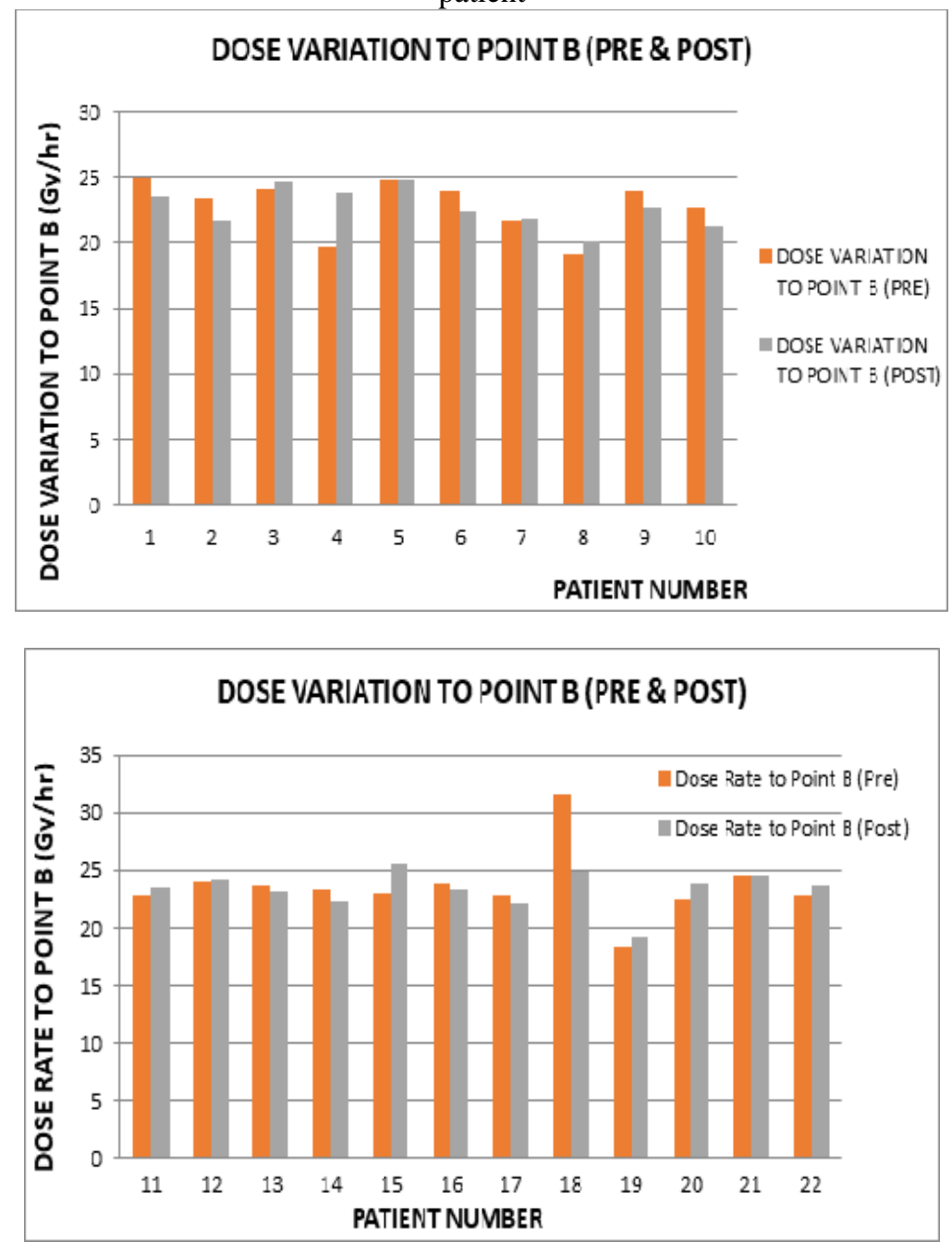

Figure 11: A bar chart of dose rate variation to point 'B' for pre \& post-treatment for the next set of 12 patients 


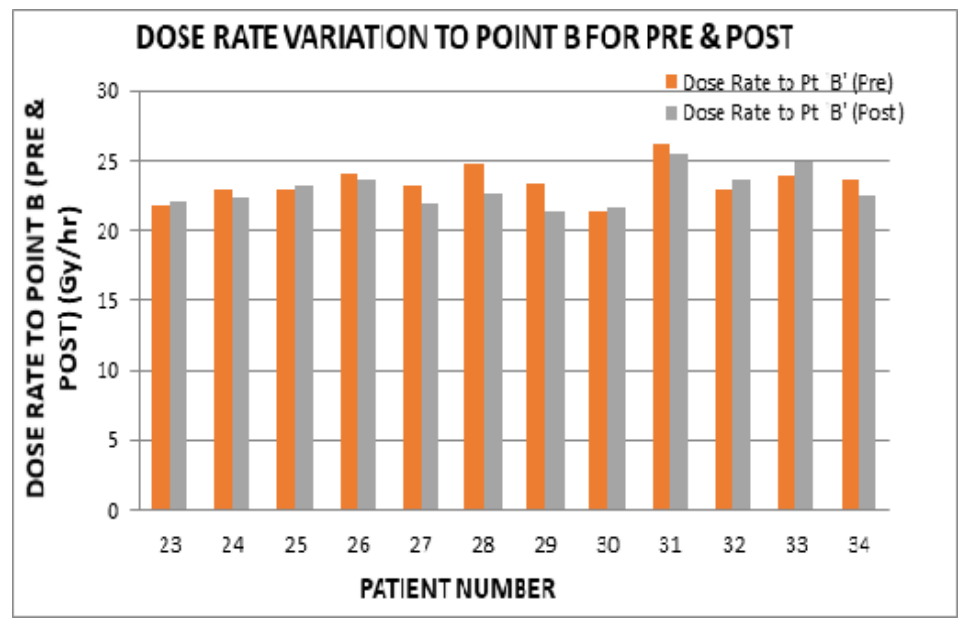

Figure 12: A bar chart of dose rate variation to point 'B' for pre \& post-treatment for the last 12 patients

The dose variation for the Bladder and Rectum are as shown below in figure $13,14,15$, and 16;

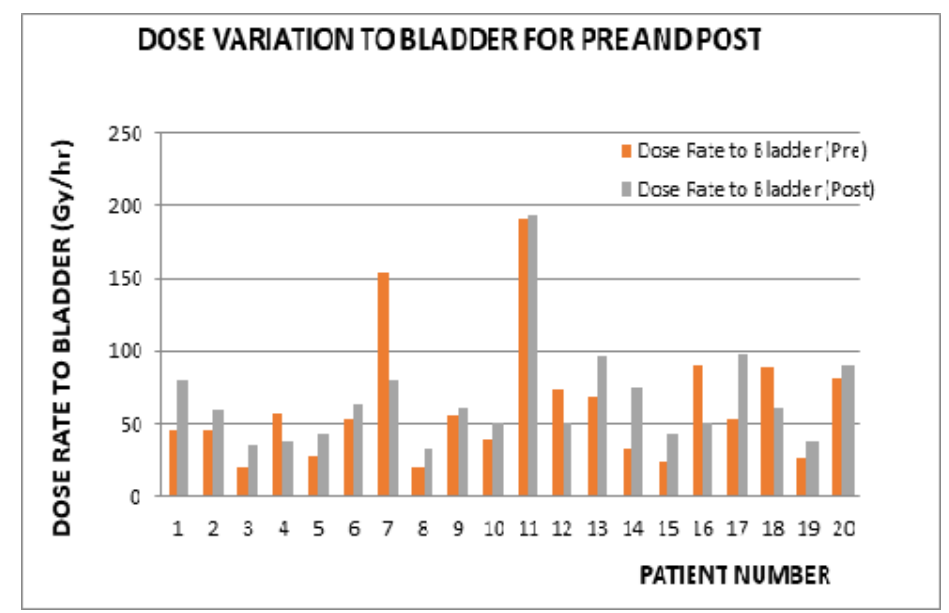

Figure 13: A bar chart of total dose received to the bladder for pre- \&post treatment calculation for the first 20 patients 


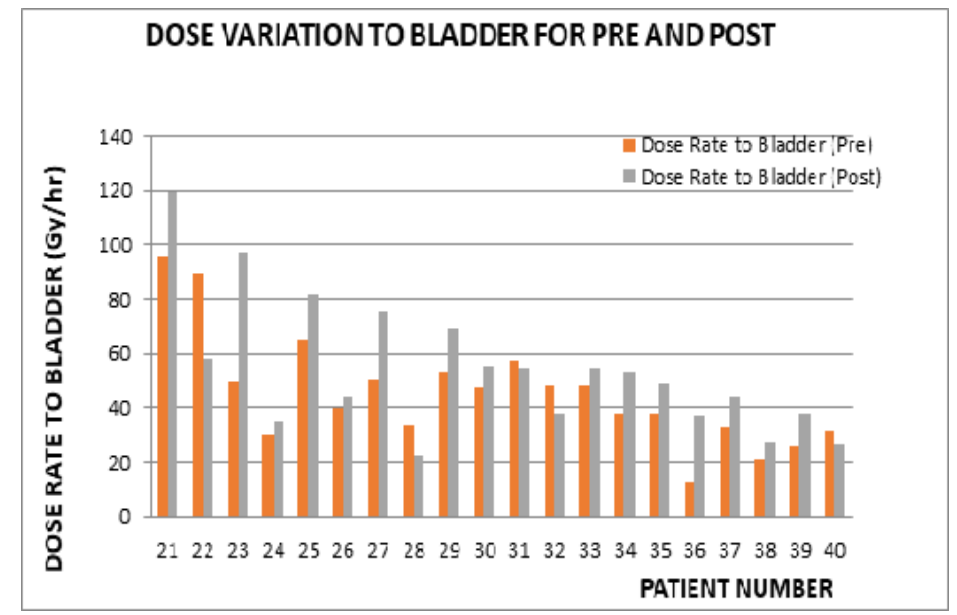

Figure 14: A bar chart of total dose received to the bladder for pre- \& post-treatment calculation for the last 20 patients

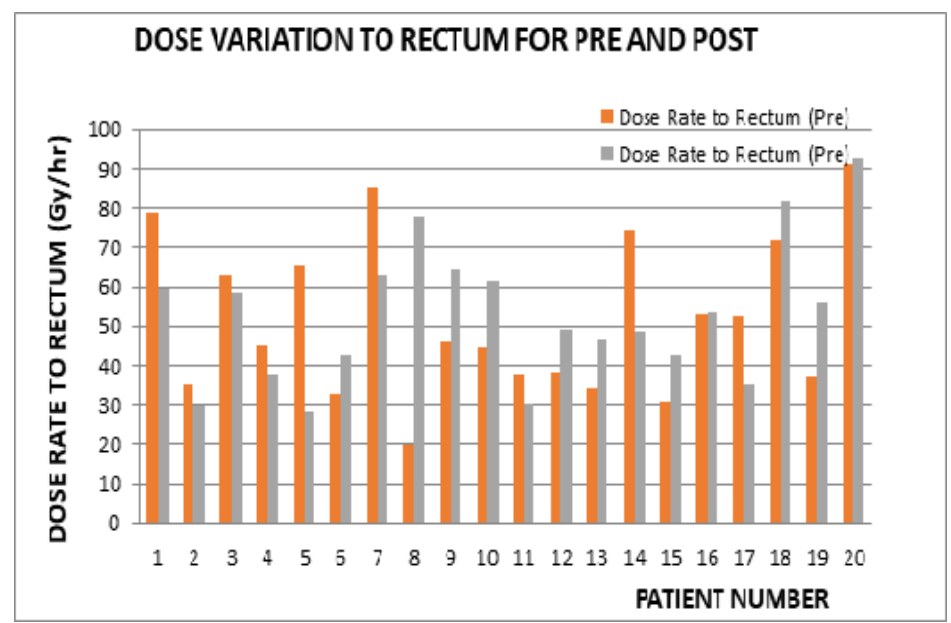

Figure 15: A bar chart of total dose received to the rectum for pre-and post-treatment calculation for the first 20 patients 


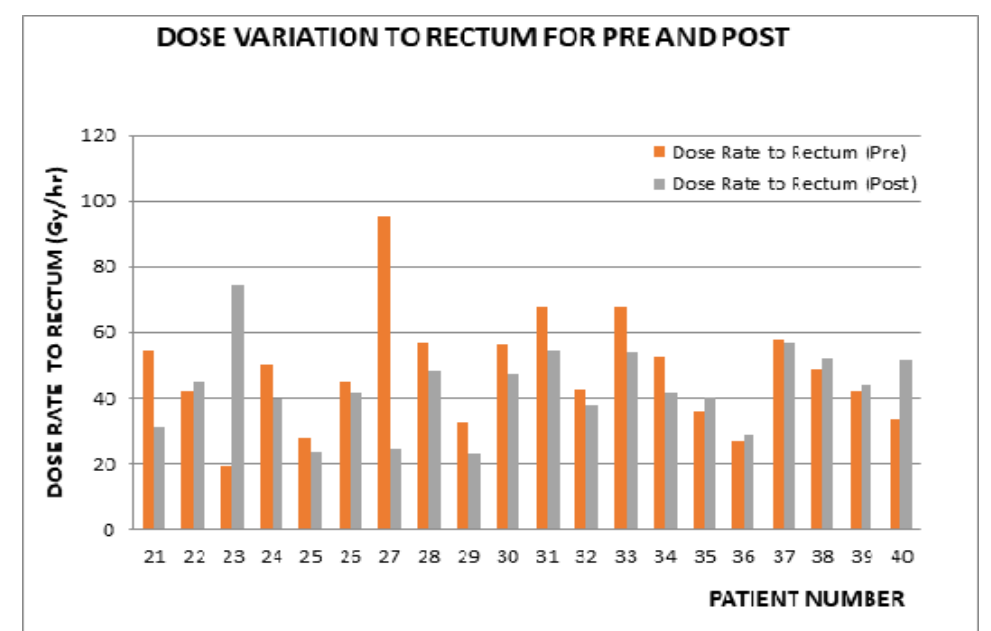

Figure 16: A bar chart of the dose received to the rectum for pre-and post-treatment calculation for the last 20 patients

\section{Discussion}

In low-dose-rate brachytherapy, clinicians prescribe doses to point 'A' since it is usually located within the tumour volume. Doses prescribed to point 'A' are usually 30 or 35Gy. The geometrical point 'A' often occurs in a high gradient region of the pear-shaped volume of isodose distribution, based on the anatomy of the patient's pelvic region in the geometrical arrangement of the radioactive source. The slightest change in position can result in significant differences in delivered dose. The minimum deviation between most of the pre-treatment calculated dose and post-treatment calculated dose for patients 2, 4, 7, 8, 15, 21, 29 and 34 showed large deviations of 125, 13, 11, 15, 13, 12, 16 and 23\%, respectively. The deviations occurred due to loose packing of the applicators, patient transport from the simulation room to the brachytherapy room, and instability of patients during the treatment. Patient 2 showed an outrageous deviation, with more than $100 \%$ dose increment. It was observed that the tandem turned to the opposite direction, making it move closer to the point 'A', thereby doubling the dose received. Patient 19 also showed a sharp decline in both pre-and post-treatment calculated doses. This resulted from a short tandem used with only two source allocations, thereby reducing the dose rate to point ' $\mathrm{A}$ '. The dose rates to point ' $\mathrm{A}$ ' for pre-and post-treatment calculated doses do not differ much (since the time taken for treatment is shorter) for the patients who had undergone total or partial hysterectomy. For hysterectomy patients, point ' $\mathrm{A}$ ' was located in the middle of the vaginal ovoids within the source. Therefore, as the dose increases, the time also becomes shorter, resulting in less patient movement during treatment. Two patients (i.e. Patients 39 and 40) showed a $10 \%$ deviation which was the highest for the hysterectomy patients. 
High variation between doses prescribed and delivered to point 'A' was not of much concern (i.e. when it does not lead to an underdose of the tumour) since it was delivered to the tumour and not to the organs at risk. This was not of much concern since further investigations of the same patient showed no alarming increase of the doses to the organs at risk. The total bladder dose for the same patient after treatment was $65.05 \mathrm{~Gy}$, whiles that of the rectum was 69.55 Gy. QUANTEC dose limit for LDR BT for the bladder was $80 \mathrm{~Gy}$, whiles that of the rectum was $75 \mathrm{~Gy}$. Some patients also showed lesser delivered doses than prescribed which was also not of concern since it did not lead to underdosing of the tumour. The incident categories are near-misses, non-conformance and incidents. A near-miss category occurred when the error was detected after prescription and before dose delivery. Non-conformance errors occurred when the procedure varied from the standard practice. An incident error was considered when dose variation occurs and categorised into levels (minor, major and severe). Point 'B' does not rotate; hence minimal deviation was observed between the pre and post-treatment calculated doses. The dose rate was meagre compared with that of the critical organs. This was assessed to find out the difference in the lymph node area. Patients 15 and 18 showed very high deviations ( $12 \%$ and $21 \%$, respectively). This deviation was attributed to the movement of the applicators due to loosening packing.

The bladder and rectum are the critical organs of interest during a cervical brachytherapy procedure. Particular interest was given to these organs to ensure that they do not exceed the acceptable limits of radiation exposure based on the volume of that organ receiving a particular dose. The American Brachytherapy Society (ABS) has given recommendations on the acceptable levels of radiation exposure to these organs at risk (Nag et al., 2004). The quantitative analysis of the average tissue effect in the clinics (QUANTEC) has set $80 \mathrm{~Gy}$ as the bladder tolerance dose for LDR brachytherapy (Viswanathan and Thomadsen, 2009). Patients 11 and 21 have their doses calculated for both pre-and post-treatment exceeding the bladder dose limit of QUANTEC. According to Sheybani et al. (2013), filling the bladder, packing with gauze, and the patient's anatomy can lead to increased doses to the bladder. This may explain why Patients 11 and 21 had both pre and post doses being high. Patients 7, 16, and 26 also had their pre-treatment calculated doses above the tolerance level, but their post-treatment calculated doses showed a reduction of the doses (lower than the tolerance level). Pre- and post-treatment images of the patient's treatment showed that the bladder had moved further away from the applicators, which in effect reduced the dose to the bladder. On the contrary, Patients 17 and 23 had their pre-treatment calculated doses below the tolerance level. However, their post-treatment calculated doses increased the doses (higher than the tolerance level). Comparing the pre and post-treatment images of the patients showed that the bladder had moved 
closer to the applicators, which increased the dose to the bladder. Average doses of 69.26Gy and 71.09Gy were obtained for the pre-and post-treatment with an average deviation of $2.32 \%$.

The quantitative analysis of average tissue effect in the clinics (QUANTEC) has set 75Gy as the rectum tolerance dose for LDR brachytherapy (Viswanathan and Thomadsen, 2009). Patient 31 had the dose calculated for both pre and post-treatment, exceeding the rectum dose limit set by QUANTEC. According to Sheybani et al. (2013), filling the rectum with gauze and the patient's anatomy can lead to increased doses to the rectum, which may explain why Patient 31 had both pre and post doses been high. Patients 7, 14, 20, 27, and 33 also had their pre-treatment calculated doses above the tolerance level, but their post-treatment calculated doses showed a reduction of the doses (lower than the tolerance level). Comparing the pre-and posttreatment images of the patients showed that the rectum had moved further away from the applicators, which in effect reduced the dose to the rectum.

On the contrary, Patients 8, 18, 19, and 20 had their pre-treatment calculated doses below the tolerance level. However, their post-treatment calculated doses increased the doses (higher than the tolerance level). Comparing the pre and post-treatment images of the patients showed that the rectum had moved closer to the applicators, which in effect increased the dose to the rectum. An average dose of $67.02 \mathrm{~Gy}$ and $66.64 \mathrm{~Gy}$ were obtained for the pre-and posttreatment with an average deviation of $0.30 \%$.

After treatment, patients must visit the clinic after the first week, monthly, quarterly, and annually to monitor their condition.

\section{Limitations of the study}

This research was limited to developing a patient treatment record analytical platform embedded in an effective radiotherapy patient management system. Regarding data privacy, the study has already been cleared for exemption by the Kwame Nkrumah University of Science and Technology ethics committee and the Komfo Anokye Teaching Hospital's ethical board. The researchers took no particular notice of patient names on the various medical records collated for the study as the main focus was on dose analysis of patient treatment. Patient identities are duly hidden, and all clues that might trace back to the patient as an individual were cleaned from the data. Hence their identities do not appear in any report or publication of this research.

\section{Conclusion}

Applicator displacement, such as the tandem and ovoids, can substantially influence patient dosimetry and, therefore, the learning process. The geometric relationships between intracavitary brachytherapy applicators 
and the critical structures differ significantly during the brachytherapy LDR procedure. Source movement results in considerable dose alterations to the critical organs, generating an increased rate of complications that can influence the cure rates. The disparity in dose to point 'A' was found to be 1.16 $\%$ which is very laudable compared to other studies that established dose variations of 2, 35, 8, and 20\% (Corn et al., 1993). This wide range is because the studies were performed with radium, iridium, and caesium sources. Variation in dose at point 'B' in this work was $0.75 \%$, while (Corn et al., 1993) showed this variation to be $1.7 \%$. As per the guidelines, there was only one rectal point chosen by most researchers, but in this study, three rectal and bladder points were respectively used to assess rectal and bladder mobility. Studies done by Corn and Pham have found dose dissimilarities in the rectum and bladder of 3 and 10\%, respectively (Corn et al., 1993); (Pham et al., 1998). This study found average variations of 2.32 and $0.30 \%$ for the bladder and rectum, respectively. The variation found between prescribed and deposited doses was $2.11 \%$ for quality assurance purposes. It can be concluded that the findings are within an acceptable range. Hence, the practice at KATH meets international standards. When safety-critical applications are being operated, incident reporting systems are employed for many reasons. Evidence shows that event reports help determine why accidents do not even happen in the first place. Incident reporting forms often identify the obstacles that prevent bad events from turning into significant mishaps. These observations are pretty valuable. To ensure the future advantages of those precautions, analysts can use them to identify areas where extra support is required. An increase in events provides deeper insight into the proportional amounts of human "error", system failure, regulatory weakness, et cetera. It is recommended that HDR brachytherapy should be considered due to advantages such as short treatment time and dose optimisation of normal tissues over the LDR BT.

\section{Acknowledgement}

The authors are much grateful to the Komfo Anokye Teaching Hospital staff for their cooperation and support to perform this research.

\section{References:}

1. Adams, A. R., Nortey, P. A., Dortey, B. A., Asmah, R. H., \& Wiredu, E. K. (2019). Cervical Human Papillomavirus Prevalence, Genotypes, and Associated Risk Factors among Female Sex Workers in Greater Accra, Ghana. Journal of Oncology, 2019. https://doi.org/10.1155/2019/8062176

2. Adewole, I.F., Benedet, J.L., Crain, B.T. \& Follen, M.2005. Evolving a strategic approach to cervical cancer control in Africa. Gynecologic Oncology 99, S209-S212. 
3. Chaffer C. L., Weinberg R. A. (2011): A perspective on cancer cell metastasis. Science; 331: 1559-1564.

4. Corn W, Galvin D, Soffen M et al. Positional stability ofsources during LDR brachytherapy for cervical carcinoma.Int $J$ RadiatOncolBiolPhys1993; 26: 513-518.

5. Denny, L. 2010. Cervical cancer in South Africa: An overview of current status and prevention strategies. CME Feb. 28(2):70-73.

6. Domfeh A. B., WireduE. K., AdjeiA. A., Ayeh-KumiP. F. K., AdikuT. K., TetteyY., Gyasi R. K. and ArmahH. B. 2008 Cervical Human Papillomavirus Infection in Accra, Ghana. Ghana Medical JournalVolume 42, Number 2, pp $72-78$

7. Hanahan D. \& Weinberg R. (2001). The hallmarks of cancer. Cell ;100: 57-70.

8. Kohn LT, Corrigan JM, Donaldson MS. To Err Is Human: Building a Safer Health System, Vol. 627. Washington, D. C.: National Academies Press; 2000.

9. Koushik K., Bilimagga R., Rao N., JanakiM.G., Ponni A., RajeevA.G. (2010) Positional variation of applicators during low doserate intracavitary brachytherapy for cervical cancer:a prospective study. Journal Contemporary Brachytherapy 2010; 2, 3: 93-97 DOI: 10.5114/jcb.2010.16919

10. Leaver, D. \& Labonte, G. 2010. HPV and cervical cancer. Radiation therapist,

19(1):27-44

Leedy, P. D. \&Ormrod, J. E. 2005. Practical research planning \& design, 8th ed. New Jersey:Pearson.

11. Ljunggren A, Swyman D, Herby L et al. (1987)An assessment of calculated doses in intracavitary gynecological radiotherapy. MedDosim; 12: 15-17

12. Makin, M.S. \& Kamanu, C.I. 2010. Prevention and treatment of cervical cancer in Africa II Surgery in Africa-Monthly Review.

13. Nag, S., Cardenes, H., Chang, S., Das, I. J., Erickson, B., Ibbott, G. S., Varia, M. (2004). Proposed guidelines for image-based intracavitary brachytherapy for cervical carcinoma: report from Image-Guided Brachytherapy Working Group. International Journal of Radiation Oncology Biology Physics, 60(4), 1160-1172.

14. Parkin D.M., Ferlay J., Hamdi-Cherif M., Sitas F.,Thomas J.O., Wabinga H. and Whelan S.L. Cancerin Africa: Epidemiology and Prevention. 4.3 Cervix Cancer. IARC Scientific Publications 2003; No 153: Lyon: IARC Press, pp 268-276. 
15. Pham T, Chen Y, Rouby E et al. Changes in HDR tandem and ovoid applicator positions during treatment in an unfixed brachytherapy system. Radiology 1998; 206: 525-531.

16. Pollack LA, Rowland JH, Crammer C, Stefanek M: Introduction: charting the landscape of cancer survivors' health-related outcomes and care. Cancer 2009; 115: 4265-4269.

17. Sheybani A., Tennapel M. J., Sun W., Kim Y., Rockey W. M. (2013) Dose-Volume Effect of Bladder and Rectal Filling during Intracavitary High-Dose-Rate Brachytherapy. Brachytherapy 12: S11-S77

18. Viswanathan, A.N. \& Thomadsen, B. Undated. (2009) American Brachythterapy Society (ABS) cervicalcancer task group. Reviewed by: Erickson, B. Gaffney, D. Small, W. \& Hsu, I.

19. WHO (World Health Organization). 2012. "Prevention of Cervical Cancer through Screening and Using Visual Inspection with Acetic Acid (VIA) and Treatment with Cryotherapy.” WHO, Geneva.

20. WHO, 2008. World cancer report 2008. Geneva: Published by the International Agency for Research on Cancer. World Health Organization (WHO). Available: http://www.iarc.fr/en/publications/pdfs-online/wrc/2008/index.pdf. $[4 / 05 / 2010]$

21. WHO, 2020 Report on cancer: setting priorities, investing wisely and providing care for all. Geneva: World Health Organization (WHO). Licence: CC BY-NC-SA 3.0 IGO. 